\section{Comprehensive palaeontology}

\author{
J.C.W. Cope
}

The Encyclopedia of Paleontology. Edited by R.W. Fairbridge and D. Jabolonski. Pp.912. (Dowden, Hutchinson and Ross: New York. Distributed by Academic Press: New York and London, 1979.) \$90, $£ 52.50$.

THE appearance of a major reference work on palaeontology within the compass of a single volume is indeed welcome. The Encyclopedia of Paleontology contains entries from over 120 palaeontologists, mainly from the USA, but with expertise from Europe, Australasia and Canada contributing some $30 \%$ of the information. Most authors have contributed only one entry, others have supplied as many as six.

The subject entries are very wide-ranging and cover in considerable detail each group of plant and animal fossils in alphabetical order. In addition to the taxonomic treatment there is a long list of other subjects covered such as biometrics, computer applications in palaeontology, palaeoecology, population dynamics and so on, in which the state of the art has clear exposition. These two categories of entries are complemented by longer discursive entries on such topics as the history of palaeontology (divided into three separate entries, historically), evolution, extinction and other subjects that lend themselves to such treatment.

The entries are well organized and provide a wealth of accurate information; each has cross-references to other entries and, where appropriate, to entries in other volumes of the Encyclopedia of Earth Sciences. The reader is directed to further reading by generally well selected and ample references. Occasionally one feels that the author of an entry has not been sufficiently selective in citation of these; as examples of over-zealous quotation of references "Paleoecology of Inland Aquatic Environments" has 110 references and "Radiolaria" almost 120 . This surely is not helpful to the average reader seeking direction to further major references.

The references provide at least a general clue to the date of writing of the entry. Some seem to have been written about ten years ago, for although recent references are listed, no reference is made to them in the text. However, the entries in general are up to date; many articles were clearly written around 1976 and references up to 1978 occur commonly. Within the limits imposed by getting such a volume published this seems very reasonable. Where work is dated, it is largely a reflection of how rapidly some aspects of palaeontology have advanced recently.

On first glancing through the book I wondered whether it had been mis-bound were nannoplankton, nautiloids, nekton, neoteny? In fact these fears were largely unjustified as most of the above subjects appear elsewhere under other headings and are listed in the extensive index which, a quick calculation suggests, contains close on 5000 subject entries, many with several page references. The index must be criticized, though, in that it sometimes refers to words which are just mentioned in passing. Thus my search for 'neoteny' led to p.34 which merely stated that certain Cretaceous Amphibia were "pedomorphic or neotenic". The index entry for "pedomorphic" only quotes p.34 so that the reader is still none the wiser on what neoteny is, and both index entries are unnecessary. Checks revealed a number of similar superfluous entries. Misprints in the text have led to index entries; thus "Nautilioids" (sic) refers to a misprint on p.533 and is quoted by the index separately from "Nautiloids" and "Nautiloidea". "Conchostraca" get separate indexing from "Conchostracans", but the index entry "Cyclostomata" refers to both the vertebrate and bryozoan orders of that name. Clearly the index was not compiled by a palaeontologist, and its value is consequently somewhat diminished.

Although the book is extremely comprehensive in its treatment, I was surprised that scant attention had been paid to the stratigraphical use of fossils. There is an apposite entry on as there are no entries under ' $N$ ' - where

biostratigraphy by Peter SylvesterBradley (one of seven authors who have since died) - but that is all. There is no entry in the index under the word 'zone', and although some systematic entries do refer to stratigraphical value of particular fossil groups, to me this is a major omission.

The book is abundantly illustrated by both half-tone and line illustrations; the great majority of them are first class and contribute significantly to the volume. The high standard of the majority makes one aware of the number which fall short of this standard in some respects; poor reproduction mars several otherwise good figures, and widely disparate sizes and styles of lettering are distracting.

The price of $\$ 90$ (or $£ 52.50$ ) seems excessive for a single volume. At half this price the number of purchasers would probably be increased at least tenfold; but the considerable authority and wealth of information contained therein should compel all palaeontologists seriously to consider buying the book. For undergraduates it will provide valuable information and speedy reference to essential reading for essays and project work; for university libraries at least one copy is essential. Despite its faults this is a volume truly worthy of its title and one which is unlikely to be rivalled for a long time.

J.C.W. Cope is Senior Lecturer in Geology at the University College of Swansea, UK.

\section{Kinetics for chemists}

\section{C.F. Wells}

Chemical Kinetics and Transport. By P.C.

Jordan. Pp.340. (Plenum: New York and London, 1979.) £13.55.

As he states in his preface, and is implied in the title, the author adopts a new style in his treatment of kinetics. The first three chapters are concerned with the fundamentals of the transport of molecules: two deal with the kinetic theory of gases, covering the properties of a collection of gaseous molecules at equilibrium and the transport of molecules; but the third introduces an unusual note for a book on kinetics, a treatment of electrolytic conduction and diffusion. In Chapter 4, the experimental approach to kinetics begins with the determination of rate laws. This is followed by a treatment of stationary state mechanisms, photochemistry and non-stationary state mechanisms. The book is then completed by a consideration of single collisions, the theory of reaction rates and various models for collisional processes.

In its concentration on chemical kinetics from a transport viewpoint, especially in the earlier chapters, the book has a novel approach. Moreover, much important material is reserved for the problems at the end of each chapter; undergraduate students attempting to follow their more conventional lecture courses with the aid of this book might well become confused. The latter state will probably be increased by what some will regard as a cavalier treatment of elementary rate laws. In Chapter 4, complex ideas are introduced too early: for example, before single rate laws are explored, the problems of the $\mathrm{H}_{2}+\mathrm{I}_{2}$ and the $\mathrm{H}_{2}+\mathrm{Br}_{2}$ reactions are stated, and we find a discussion of nanosecond flash photolysis; or, before a discussion of simple orders higher than one, we are plunged into opposed first order reactions. Indeed, in a footnote the reader is referred to a standard text to obtain a detailed understanding of the integrated rate laws. Perhaps this is the most unsatisfactory chapter from an undergraduate point of view, as there are also minor contributions to his confusion. For example, in the section on first order reactions, one finds a jumble of first order and opposed first order reactions; or, on p.90, it is stated that the first term in eqn 4.31 vanishes, whereas, in the balance of rates at equilibrium, the first two terms disappear. However, if the student survives this chapter, or is assisted 